\title{
Relación especie-área y distribución de la abundancia de especies en una comunidad vegetal de un inselberg tropical: efecto del tamaño de los parches
}

\author{
Pedro Manuel Villa1,2,3*, Lucas de Siqueira Cardinelli4, Luiz Fernando Magnago ${ }^{5}$, \\ Gustavo Heringer ${ }^{1,3,6}$, Sebastião Venâncio Martins ${ }^{1,2}$, Prímula Viana Campos ${ }^{1}$, \\ Alice Cristina Rodrigues ${ }^{1,3,6}$, Andreza Viana Neri ${ }^{1,6}$ \& João Augusto Alves Meira-Neto ${ }^{1,6}$ \\ 1. Universidade Federal de Viçosa, Programa de Pós-Graduação em Botânica, 36570-900, Viçosa, Minas Gerais, Brasil; \\ pedro.villa@ufv.br, primula_v@yahoo.com.br \\ 2. Universidade Federal de Viçosa, Departamento de Engenharia Florestal, Laboratório de Restauração Florestal, 36570- \\ 900, Viçosa, Minas Gerais, Brasil; venancio@ufv.br \\ 3. Fundación para la Conservación de la Biodiversidad, 7101, Puerto Ayacucho, Amazonas, Venezuela; \\ gustavoheringer@hotmail.com, alicecristina261@gmail.com \\ 4. Instituto de Botânica, 4301902, São Paulo, São Paulo, Brasil; lucasscardinelli@hotmail.com \\ 5. Universidade Estadual de Santa Cruz, 45662-900, Ilhéus, Bahia, Brasil; luiz_fsm@hotmail.com \\ 6. Universidade Federal de Viçosa, Departamento de Biologia Vegetal, Laboratório de Ecologia e Evolução de Plantas, \\ 36570-900, Viçosa, Minas Gerais, Brasil; andreza.neri@ufv.br; j.meira@ufv.br \\ * Correspondencia
}

Recibido 14-III-2018. C Corregido 23-III-2018. Aceptado 05-IV-2018.

\begin{abstract}
Species-area relation and species abundance distribution in a plant community on a tropical inselberg: effect of patch size. Although inselbergs are iconic rock outcrops with a high biogeographic value, little is known about drivers responsible for the plant community assembly. The aim of this research was to evaluate how the patch size distribution of vegetation influences the species-area relationship and species abundance distribution of a community in an inselberg of the "Piedra La Tortuga" Natural Monument of the Guayana region, Venezuela. In this context, three research questions were established: What is the effect of patch size on species richness? What species-area model (SAR) has the best fit in those vegetation patches? How is the distribution of species abundances (SADs) induced by the patch size distribution? A stratified random sampling was performed in patches ranging from 0.34 to $14.8 \mathrm{~m}^{2}$, totaling 40 sampling units $\left(226 \mathrm{~m}^{2}\right)$. All individuals found in the 40 patches were identified at species level. The floristic composition in the different samples was represented by 19 families, 22 genera and 24 species, of which $50 \%$ are endemic to inselbergs and two, are threatened of extinction. Two groups of patch sizes were identified (large 8-15 $\mathrm{m}^{2}$ and small $\leq 7.9 \mathrm{~m}^{2}$ ) in relation to the abundance and composition of species. The species accumulation curves for each patch size group show a contrasting tendency with marked differences in the observed richness among patch size groups. The curves of the SADs models had a significant adjustment of the geometric series in the two categories of patches. The SAR model of the power function presented the best species-area adjustments, where the increase in patch area accounted for $82 \%$ of the variation in the increase in the number of species. The results of this study demonstrate for the first time how vegetation patches of a tropical inselberg have a strong influence on richness, abundance distribution and species composition. Likewise, it was determined that the SAD geometric model presented the best fit in the community as a function of patch size as a resource indicator, where the abundance of a species can be equivalent to a proportion of the space occupied. It is also presumed that changes in patch sizes could be associated with nutrient and water availability, as has been demonstrated in other dryland environments. In some studies it has been argued that variation in species composition among vegetation profiles of tropical inselbergs is mainly conditioned by habitat structure and water deficit. However, it had not been discussed how the size of patches of vegetation has an effect on richness. SADs and SAR analyzes can provide complementary explanations on community assembly in inselbergs. Rev. Biol. Trop. 66(2): 937-951. Epub 2018 June 01.
\end{abstract}

Key words: community assembly; geometric series; power law; rarefaction; richness; vegetation patches. 
Los inselbergs tropicales son afloramientos rocosos generalmente de granito que emergen abruptamente de sus ecosistemas circundantes (sabanas, bosques), configurándose como islas terrestres aisladas (Porembski, 2000; Porembski \& Barthlott, 2000). Las características geomorfológicas y microclimáticas de estos afloramientos confieren condiciones ecológicas extremas en los hábitats donde se desarrollan especies con distribución restringida y endémica (Barthlott, Porembski, Szarzynski, \& Mund, 1993; Gröger, 2000; Porembski, 2007), principalmente en los mosaicos de parches que se consideran islas de vegetación sobre las islas de rocas (Porembski, 2000; Porembski, 2007). Por lo tanto, las reglas de estructuración de las comunidades vegetales (Assembly rules, definición en inglés) de los inselbergs tropicales están fuertemente influenciadas por las condiciones ambientales extremas, principalmente por el efecto de la escasez de agua y nutrientes, así como de los suelos poco profundos y elevada temperatura microclimática (Lüttge, 1997; Gröger \& Huber, 2007). En este sentido, los parches de vegetación aislados en las depresiones, configurando un mosaico discontinuo, representan unas de las comunidades más resaltantes de los inselbergs tropicales con las condiciones ambientales más extremas (Porembski, 2007). A pesar del amplio conocimiento existente sobre patrones fitogeográficos de los inselbergs, todavía existen limitaciones en el entendimiento sobre la estructuración de las comunidades vegetales inducidas por las características de los tipos de hábitats.

El análisis de la distribución de los tamaños de parches de vegetación ha sido utilizado para evaluar patrones de diversidad y estructuración de comunidades vegetales donde el agua es el principal filtro ambiental (Svejcar, Bestelmeyer, Duniway, \& James, 2015; Meloni, Granzotti, Bautista, \& Martinez, 2017). De esta forma, datos empíricos y modelos teóricos indican que los tamaños de parches de vegetación en tierras secas (drylands, término en inglés) pueden variar de forma aleatoria sin escala definida en función de la heterogeneidad ambiental (Moreno de Las Heras, Saco,
Willgoose, \& Tongway, 2011; Barbier, Bellot, Couteron, Parsons, \& Mueller, 2014; Meloni et al., 2017). Sin embargo, existe limitada información que explique cómo el tamaño de los parches en este tipo de ambientes influye directamente en la estructuración de las comunidades. Un método clásico para entender la estructuración de comunidades vegetales, ha sido considerar el espacio como un recurso que se divide en puntos discretos, los cuales son ocupados por un individuo dentro de la comunidad (Tilman, 1994; Götzenberger et al., 2012). Por este motivo, una forma indirecta de evaluar la interacción de las especies con la disponibilidad de recursos del ambiente (agua, luz, nutrientes) es analizando la relación dependiente de la escala del recurso espacio con la presencia y abundancia de las especies (Amarasekare, 2003; Rietkerk \& van de Koppel, 2008; Götzenberger et al., 2012). Por lo tanto, las especies dominantes presentan mayor capacidad de ocupar una mayor amplitud del espacio en relación a las restringidas localmente con requerimientos ambientales específicos del gradiente (Götzenberger et al., 2012), lo que podría ser analizado parcialmente para cada sitio por medio de la relación dominancia-diversidad, basado en la distribución de las abundancias de todas las especies de la comunidad.

La distribución de abundancia de especies (SADs, por sus siglas en inglés) caracteriza la distribución de abundancias de todas las especies dentro de una muestra o comunidad ecológica, observándose como patrón general que la mayoría de las especies son relativamente raras y sólo algunas son dominantes (McGill et al., 2007; Borda de Água, Borges, Hubbell, \& Pereira, 2012). Entre los modelos SADs, el modelo de la serie geométrica explica adecuadamente el patrón de distribución de abundancia de las especies en función de la partición proporcional de recursos (tamaño del parche como indicador del recurso espacio), donde la abundancia de una especie puede ser equivalente a la porción del espacio ocupado por ella (Magurran, 2004; Alroy, 2015). Los parámetros generados en cada modelo SAD particular son dados por la estructura de las distribuciones 
espaciales de las especies, luego un modelo SAD puede explicar la distribución del recurso espacio entre los hábitats (Sizling, Storch, Sizlingová, Reif, \& Gaston, 2009; Matthews \& Whittaker, 2015). El modelo de la serie geométrica representa una mayor proporción de especies dominantes y una menor proporción de especies raras (distribución no uniforme), y ha sido encontrado para comunidades en áreas restringidas con ambientes extremos (Magurran, 2004; Fattorini, Rigal, Cardoso, \& Borges, 2016). Por otro lado, los resultados de investigaciones realizadas en Drylands también han encontrado la prevalencia de SADs lognormal en ambientes heterogéneos, permitiendo comunidades de plantas más uniformemente distribuidas (Ulrich, Soliveresb, Thomasc, Dougilld, \& Maestre, 2016).

El incremento del número de especies observadas en función del área muestreada es una de las relaciones ecológicas más conocidas (Rosenzweig, 1995), desde investigaciones pioneras de la relación especie-área (SARs, por sus siglas en inglés) en comunidades vegetales realizadas por Arrhenius (1921) y Gleason (1922). Arrhenius (1921) presentó el principal modelo utilizado para describir la relación entre el número de especies (S) y el área (A), a través de la función potencia $\mathrm{S}=\mathrm{cA}^{\mathrm{z}}$ (powerlaw en inglés). En este modelo el parámetro c corresponde a la riqueza de especies en función del área, mientras que z corresponde a la inclinación de la curva en el espacio log-log. De este manera, el efecto de la dependencia de la escala en la diversidad ha sido muy importante para entender patrones de distribución y composición de especies; considerando que es bien aceptado que los parámetros SAR pueden ser diferentes entre tipos de comunidades vegetales y escalas espaciales (Crawley \& Harral, 2001; Pan, 2013; Matthews et al., 2016).

El objetivo de esta investigación fue evaluar cómo el tamaño de los parches de vegetación influye en la relación especie-área y distribución de la abundancia de especies de una comunidad en un inselberg del Monumento Natural "Piedra La Tortuga", estado Amazonas, Venezuela. Esta premisa lleva a establecer tres preguntas básicas de partida respecto a las comunidades vegetales de los parches de este inselberg: 1) ¿Cuál es el efecto del tamaño de los parches sobre la riqueza de especies? 2) ¿Qué modelo SAR presenta mejor ajuste en esos parches de vegetación? 3) ¿Cómo la distribución de la abundancia de especies es inducida por el tamaño de los parches? Además, se espera encontrar un patrón de segregación de abundancia entre tamaños de parches por el modelo SADs de la serie geométrica, por tratarse de comunidades presentes en áreas restringidas con ambientes extremos. Cabe destacar que el Monumento Natural "Piedra La Tortuga" como parte de un importante centro de endemismo (Huber, 1995), es uno de los pocos inselbergs que hacen parte de un área protegida en toda la región de Guayana. Por otra parte, es la primera vez que se evalúan los modelos SAR y SAD para un inselberg tropical. Estos modelos han sido considerados como herramientas valiosas para analizar patrones de diversidad en comunidades vegetales (Tjørve, 2012; Matthews \& Whittaker, 2015).

\section{MATERIALES Y MÉTODOS}

Área de estudio: La investigación fue realizada en el Monumento Natural "Piedra La Tortuga" ubicado al margen derecho del río Orinoco (5³3'46”'N \& 67³5'34" W, $90 \mathrm{~m}$ de altitud) municipio Átures, estado Amazonas, Venezuela. Este monumento es un afloramiento rocoso de granito que se encuentra dentro de una matriz de bosque seco tropical característico de la región Guayana, con un alto valor biogeográfico (Gröger, 1994; Esteves \& Dumith, 1997). El área de estudio presenta una periodicidad acentuada de la precipitación, donde los mayores regímenes se distribuyen entre mayo y agosto, y un período marcadamente seco entre diciembre y marzo. La precipitación media anual es de $2218 \mathrm{~mm}$, y la temperatura media de $26{ }^{\circ} \mathrm{C}$ aproximadamente (Gröger \& Hubber, 2007). En un levantamiento florístico pionero en el afloramiento rocoso "Piedra La Tortuga" se identificaron cuatro principales tipos de comunidades vegetales, i) epilíticas sobre 
roca descubierta, ii) casmofítica entre bloques monolíticos de rocas, iii) parches aislados en las depresiones, iv) bosques bajos y continuas en la meseta y bajío (Gröger, 1994).

Muestreo de vegetación: El muestreo se realizó en los parches de vegetación de las depresiones del afloramiento rocoso durante el período de mayor precipitación entre los meses de junio y julio de 2008, para incluir en el muestreo las especies efímeras que no están presentes durante el período seco. Se realizó un muestreo aleatorio estratificado en parches que oscilaron entre 0.34 y $14.8 \mathrm{~m}^{2}$, totalizando 40 unidades muestrales $\left(226 \mathrm{~m}^{2}\right)$. Se recolectaron muestras botánicas de todas las especies asociadas a los parches seleccionados para una posterior clasificación taxonómica y por formas de vida en el herbario Julian Steyermark de la ciudad de Puerto Ayacucho. Se contabilizaron todos los individuos de cada especie en cada categoría de parche con la finalidad de cuantificar la abundancia absoluta (número total de individuos de cada especie).

Análisis de los datos: Los parches fueron ordenados por medio de un análisis multivariado de escalonamiento multidimensional no métrico (NMDS) basado en el índice de similitud de Bray-Curtis usando las abundancias de las especies de las 40 unidades muestrales. Posteriormente, se realizó un análisis multivariado de varianza no paramétrica (Permanova de una vía, con 999 iteraciones) para verificar diferencias entre los grupos de tamaños definidos por el NMDS. Para comparar la riqueza de especies observadas entre los grupos de tamaños de parches, se construyeron curvas de acumulación de especies con su respectivo intervalo de confianza (95\%), haciendo ajustes de rarefacción y extrapolación basado en el área de los parches y en el número de individuos. Las extrapolaciones se realizaron considerando los datos de presencia-ausencia (número de Hill de orden 0), y la extrapolación fue hasta tres veces el tamaño de la muestra (Colwell et al., 2012). Se estimó la riqueza máxima de especies para cada categoría de parches utilizando el estimador no paramétrico de Chao 1, que es menos tendencioso para muestras pequeñas.

Se analizaron modelos SADs a través de la construcción de curvas de dominancia-diversidad como método para clasificar las abundancias de las especies en los parches del inselberg de forma decreciente, desde las especies más abundantes hasta las menos abundantes (Magurram, 2004). Cada especie presenta un rango en el eje " $x$ ", y su respectiva abundancia sobre el eje "y". Existen diferentes tipos de modelos SADs para representar curvas de rango de especies (Species rank en inglés), por lo que el primer paso consistió en la identificación y evaluación del nivel de ajuste de cuatro conocidos modelos potenciales aplicado en comunidades vegetales, el geométrico, lognormal, zipf y mandelbrot (Ulrich, Ollik, \& Ugland, 2010). Los modelos SADs se eligieron a partir de la máxima verosimilitud según el criterio de información de Akaiake (AIC), donde el menor valor de AIC indica el mejor ajuste de las curvas entre los modelos comparados (Oksanen et al., 2017). Para el análisis SAD, se eligió el modelo de la serie geométrica (Preemtion por su traducción en inglés) por presentar el mejor ajuste, y las curvas de los rangos de especies se ajustaron a regresiones lineales.

Las relaciones especie-área (SARs) se probaron mediante la aplicación de dos tipos de modelos de regresión, el modelo power-law, según lo descrito por Arrhenius $\left(\mathrm{S}=\mathrm{cA}^{\mathrm{z}}\right)$, y el modelo exponencial o semi-log, según lo descrito por Gleason ( $\mathrm{S}=\mathrm{c}+\mathrm{zlog}(\mathrm{A})$ ) (Tjørve \& Tjørve, 2008; Tjørve, 2012). En estas ecuaciones, $\mathrm{S}$ expresa el número de especies, A indica el área, c y z son las constantes intercepto y pendiente respectivamente, que se obtienen por análisis de regresión. El modelo de potencia fue evaluado en su forma logarítmica y su linealización se representa como $\operatorname{logs}=\log \mathrm{c}+$ zlogA (Tjørve \& Tjørve, 2008; Tjørve, 2012). Se aplicó un análisis de covarianza (ANCOVA), evaluando la riqueza de especies (variable respuesta) de las 40 unidades muéstrales, en función del área de los parches como variable explicativa continua, y la distribución de tamaños de parches como una variable explicativa 
categórica con dos niveles (parches pequeños y grandes). Este análisis se hizo con los datos logaritmizados para mantener homocedasticidad. El ajuste de la relación especie-área con el modelo power law y el modelo exponencial fue evaluado a través de la comparación de los valores $\mathrm{z}$, debido a que proporcionan informaciones sobre la pendiente de la relación entre riqueza de especies y área, su significancia estadística (valores $\mathrm{p}$ ) y respectivos valores de $\mathrm{r}^{2}$ (Tjørve, 2009).

Antes de realizar los análisis estadísticos se evaluó la normalidad y distribución de los datos de las variables a través de la prueba de Shapiro-Wilk. Se utilizaron pruebas de Kruskal-Wallis para comparar la riqueza y valores z (inclinación del modelo) (Tjørve \& Tjørve, 2008) de los análisis SAR entre tamaños de parches. Para el análisis NMDS se utilizó la función metaMDS, y para el análisis SAD se utilizó la función radfit del paquete 'vegan' (Oksanen et al., 2017). Las curvas de rarefacción y extrapolación fueron creadas a través del paquete 'iNEXT' (Chao et al., 2014). Se aplicó el paquete $m m S A R$ para el modelado de la relación especie-área (Guillaumon, Mouillot,
\& Gimenez, 2010). Todos los análisis fueron realizados usando el software estadístico $\mathrm{R}(\mathrm{R}$ Core Team Development, 2016).

\section{RESULTADOS}

La composición florística en las diferentes muestras estuvo representada por 19 familias, 22 géneros y 24 especies (Cuadro 1). Las familias con más géneros dentro del área de estudio fueron las Araceae con tres géneros, seguido por Fabaceae, Bromeliaceae y Melastomataceae, con dos géneros respectivamente. Los géneros con más especies fueron Caladium y Pitcairnia. Se identificaron 12 especies endémicas de inselbergs; es decir un $50 \%$ del total de las especies muestreadas en la comunidad. Las formas de vida dominantes de la comunidad fueron las fanerófitas y terófitas (Cuadro 1).

Con el NMDS se identificaron dos grupos de tamaños de parches (grandes $8-15 \mathrm{~m}^{2}$ y pequeños $\leq 7,9 \mathrm{~m}^{2}$ ) en relación a la abundancia y composición de especies, con diferencias significativas entre los grupos (Permanova, $\mathrm{P}$ $<0.001, \mathrm{~F}_{1,39}=24.54$; stress $=0.105$, Fig. 1A). En el MNDS se observó que los parches
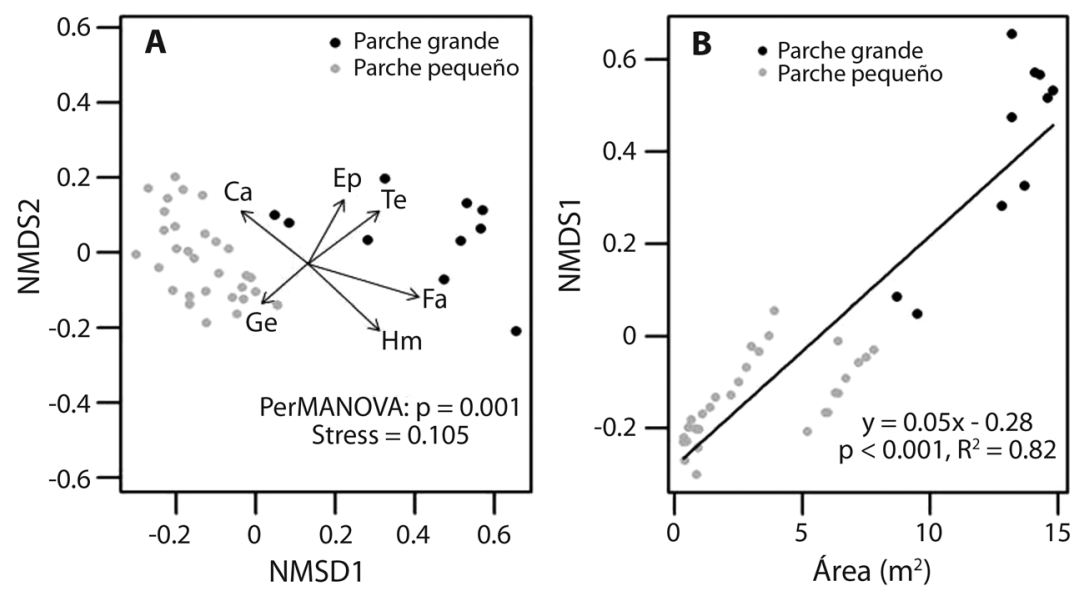

Fig. 1. Ordenación con escalonamiento multidimensional no métrico (NMDS) basado en el índice de disimilaridad de BrayCurtis usando las abundancias de las especies (A). Los vectores indican correlación de las formas de vida entre categorías de parches: Hemicriptófitas (He), Nanofanerofitas (Fa), Caméfitas (Ca), Geófitas (Cg), Terófitas (Te), Epífitas (Ep). Relación entre valores del eje 1 de NMDS y el área de los parches (B).

Fig. 1. Non-metric multidimensional scaling (NMDS) based on dissimilar Bray-Curtis using species abundances (A). The vectors indicate correlation of life forms between categories of patches: Hemicryptophytes (He); Nanofanerofitas (Fa); Camphytes (Ca); Geophytes (Cg); Terophytes (Te); Epiphytes (Ep). Relationship between NMDS axis 1 scores and patch area (B). 
CUADRO 1

Lista de especies muestreadas identificando sus respectivas formas de vida (Fv)

TABLE 1

List of species with their respective life forms (Fv)

\begin{tabular}{|c|c|c|c|c|}
\hline Familia & Especie & $\mathrm{FV}$ & $\mathrm{Pp}$ & $\mathrm{Pg}$ \\
\hline Apocynaceae & Mandevilla lancifolia Woodson* & $\mathrm{Cg}$ & $\mathrm{x}$ & $\mathrm{x}$ \\
\hline \multirow[t]{3}{*}{ Araceae } & Anthurium bonplandii G.S. Bunting & $\mathrm{Cm}$ & & $\mathrm{x}$ \\
\hline & Caladium macrotites Schott & Ep & & $\mathrm{x}$ \\
\hline & Caladium picturatum $\mathrm{K}$. Koch \& Bouché & Ep & & $\mathrm{x}$ \\
\hline Bignoniaceae & Tabebuia orinocensis (Sandwith) A.H. Gentry* & $\mathrm{Fa}$ & $\mathrm{x}$ & $\mathrm{x}$ \\
\hline \multirow[t]{2}{*}{ Bromeliaceae } & Pitcairnia armata Maury* & $\mathrm{Hm}$ & $\mathrm{x}$ & $\mathrm{x}$ \\
\hline & Pitcairnia pruinosa Kunth* & $\mathrm{Hm}$ & $\mathrm{x}$ & $\mathrm{x}$ \\
\hline Burseraceae & Commiphora leptophloeos (Mart.) J.B. Gillett & $\mathrm{Fa}$ & & $\mathrm{x}$ \\
\hline Cactaceae & Melocactus mazelianus Ř́ha* & $\mathrm{Ca}$ & $\mathrm{x}$ & $\mathrm{x}$ \\
\hline Clusiaceae & Oedematopus obovatus (Spruce ex Planch. \& Triana) & $\mathrm{Fa}$ & & $\mathrm{x}$ \\
\hline Cyperaceae & Bulbostylis leucostachya (Kunth) C.B. Clarke* & $\mathrm{Te}$ & $\mathrm{x}$ & $\mathrm{x}$ \\
\hline \multirow[t]{3}{*}{ Fabaceae } & Centrosema brasilianum (L.) Benth. & $\mathrm{Ca}$ & & $\mathrm{x}$ \\
\hline & Desmodium adscendens var. orinocense DC.* & $\mathrm{Hm}$ & $\mathrm{x}$ & $\mathrm{x}$ \\
\hline & Mimosa microcephala Humb. \& Bonpl. ex Willd. & $\mathrm{Fa}$ & & $\mathrm{x}$ \\
\hline Gentineaceae & Chelonanthus alatus (Aubl.) Pulle & $\mathrm{Te}$ & $\mathrm{x}$ & $\mathrm{x}$ \\
\hline Iridaceae & Cipura rupicola Goldblatt \& Henrich* & $\mathrm{Ca}$ & $\mathrm{x}$ & $\mathrm{x}$ \\
\hline Lentibulariaceae & Utricularia subulata L. & $\mathrm{Te}$ & & $\mathrm{x}$ \\
\hline Malvaceae & Pseudobombax croizatii A. Robyns* & $\mathrm{Fa}$ & $\mathrm{x}$ & $\mathrm{x}$ \\
\hline \multirow[t]{2}{*}{ Melastomataceae } & Acanthella sprucei Benth. \& Hook. f.* & $\mathrm{Fa}$ & $\mathrm{x}$ & $\mathrm{x}$ \\
\hline & Pterogastra minor Naudin & $\mathrm{Te}$ & & $\mathrm{x}$ \\
\hline Ochnaceae & Sauvagesia ramosissima Spruce ex Eichler & $\mathrm{Te}$ & & $\mathrm{x}$ \\
\hline Poaceae & Thrasya paspaloides S. Denham* & $\mathrm{Te}$ & & $\mathrm{x}$ \\
\hline Portulacaceae & Portulaca pygmaea Steyerm.* & $\mathrm{Cg}$ & $\mathrm{x}$ & $\mathrm{x}$ \\
\hline Rubiaceae & Borreria pygmaea Spruce ex K. Schum.* & $\mathrm{Te}$ & & $\mathrm{x}$ \\
\hline
\end{tabular}

Hemicriptófitas (Hm), Fanerófitas (Fa), Camellos (Ca), Geófitas (Cg), Terófitas (Te), Epífitas (Ep). Se indican las especies en las diferentes categorías de tamaños de parches $(\mathrm{x})$, pequeños $(\mathrm{Pp})$ y grandes $(\mathrm{Pg})$, y las especies endémicas $(*)$.

Hemicryptophytes (Hm), Phanerophytes (Fa), Camephytes (Ca), Geophytes (Cg), Terophytes (Te), Epiphytes (Ep). Species in different patch sizes categories $(\mathrm{x})$, small $(\mathrm{Pp})$ and large $(\mathrm{Pg})$, and endemic species $(*)$ are indicated.

pequeños están relacionados con las formas de vida camitas y geófitas, mientras que los parches más grandes con fanerófitas y terófitas. En el caso de la distribución de las abundancias por formas de vida, se encontró una alta correlación con los tamaños de parches, explicando entre $60 \%$ y $90 \%$ de la varianza. La estructura de la comunidad, representada por el primer eje del NMDS, estuvo fuertemente correlacionada con el área cuando fue analizado por grupos de tamaños de parches (Fig. 1B).
Las curvas de acumulación de especies para cada grupo de tamaño de parche muestran una tendencia contrastante con marcadas diferencias en la riqueza observada entre los grupos de tamaños de parches $(\mathrm{H}=19.71$, df $=1, \mathrm{P}<$ 0.0001). Por otra parte, se observan valores similares entre la riqueza observada y estimada en cada categoría de parche (Fig. 2). Cuando se analizan las curvas de rarefacción por categoría, se observa que los parches pequeños tienden a estabilizarse aproximadamente en un 

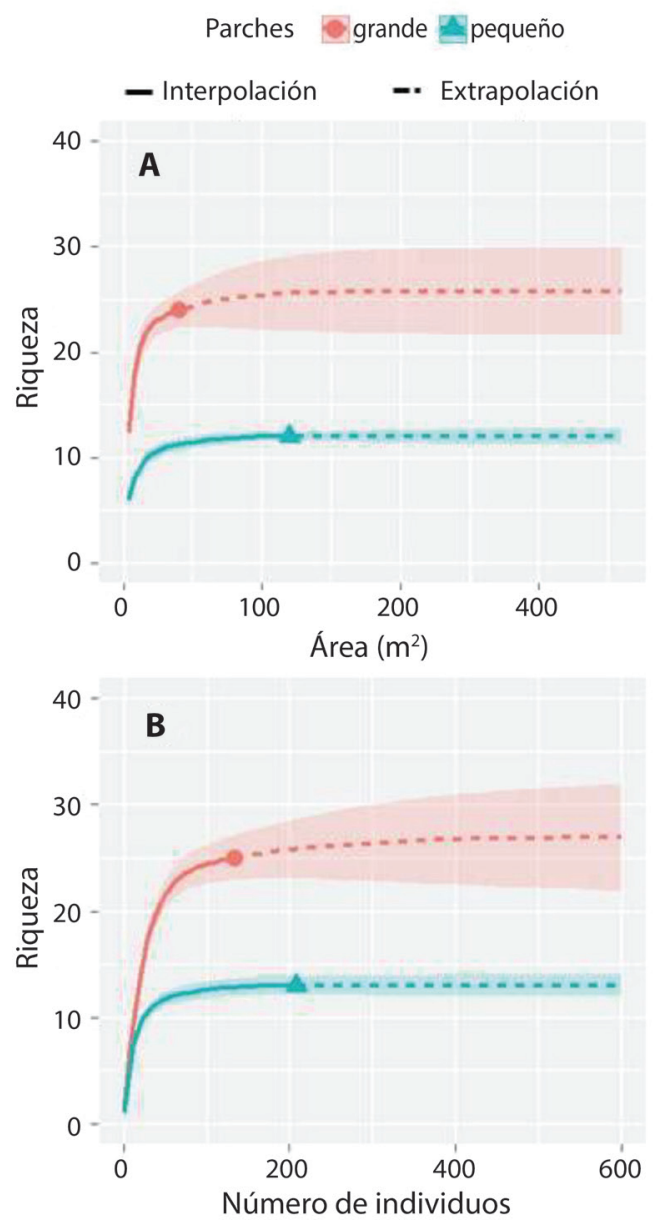

Fig. 2. Curvas de acumulación de especies por unidad de área para los dos grupos de parches (Grande $=126 \mathrm{~m}^{2}$, pequeño $=48 \mathrm{~m}^{2}$ ). Las líneas continuas con símbolos representan las curvas de rarefacción con valores observados de riqueza, las líneas discontinuas representan la extrapolación, y las áreas sombreadas en cada curva son el intervalo de confianza del $95 \%$. Se indica la riqueza observada (grande $=24$, pequeño $=12$ ) y riqueza estimada con Chaol $($ grande $=24.4$, pequeño $=12.7$ ).

Fig. 2. Species accumulation curves based per unit area for the two groups of patches $\left(\right.$ Grande $=126 \mathrm{~m}^{2}$, small $=48$ $\mathrm{m}^{2}$ ). Continuous lines with symbols represent rarefaction curves with observed richness values, dashed lines represent extrapolation, and the shaded areas in each curve are the $95 \%$ confidence interval. The observed richness (large $=24$, small $=12)$ and estimated richness with Chao1 (large $=24.4$, small $=12.7)$ are indicated . área de $50 \mathrm{~m}^{2}$ (100 individuos), mientras que los parches grandes se estabilizan con la extrapolación aproximadamente con $100 \mathrm{~m}^{2}$ de área muestreada (200 individuos).

Las curvas de los modelos SADs tuvieron un ajuste significativo de la serie geométrica en las dos categorías de parches. Estos modelos de la serie geométrica presentaron un buen ajuste en las dos categorías de parches indicando que podrían explicar adecuadamente el patrón de distribución de las abundancias (Fig. 3). A pesar de las diferencias en la riqueza específica entre los dos grupos, la distribución de las abundancias mantiene el mismo patrón, donde pocas especies son las responsables del mayor número de individuos dentro de la comunidad. Las especies más abundantes, presentes en todos los tamaños de parches fueron Tabebuia orinocensis, Pitcairnia armata, Mandevilla lancifolia y Bulbostylis leucostachya, que representaron entre el 40 y el $55 \%$ del total de la abundancia proporcional en las dos categorías de parches.

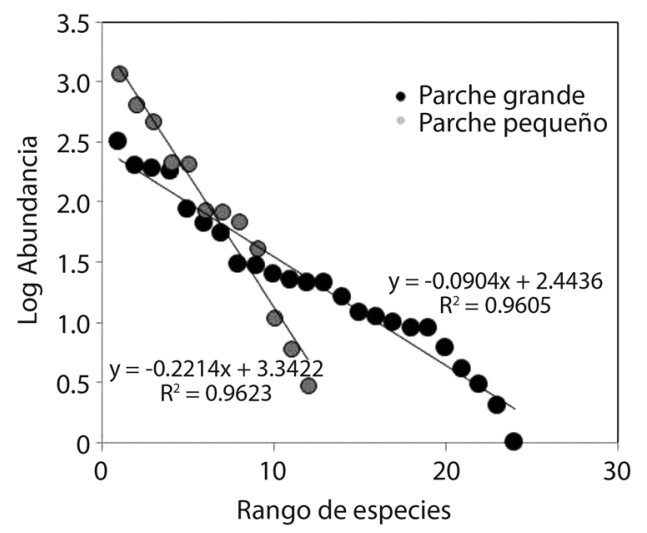

Fig. 3. Distribución de abundancias de las especies en los dos grupos de tamaños de parches, considerando la abundancia relativa en los parches grandes $(n=10)$ y pequeños $(n=30)$.

Fig. 3. Species abundance distribution in both patch sizes, considering the relative abundance in large $(n=10)$ and small patches $(\mathrm{n}=30)$. 


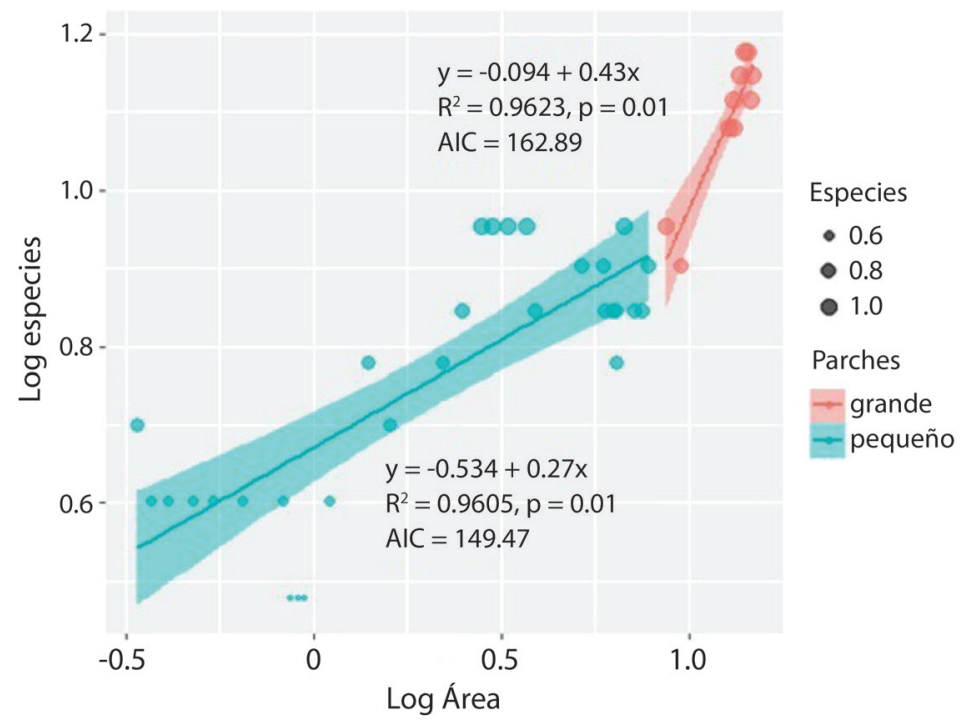

Fig. 4. Relación especie-área presentado como log-log con estimación lineal (métodos de mínimos cuadrados). Se indican los valores de la pendiente (z), la constante (C), el coeficiente de regresión, la significación estadística, y el AIC del modelo de regresión $(\log \mathrm{S}=\log \mathrm{c}+\mathrm{z} \log \mathrm{A})$.

Fig. 4. Species-area relationship presented as log-log with linear estimation (least squares methods). The slope (z), constant (c), regression coefficient, significance valeu, and AIC of the regression model $(\log \mathrm{S}=\log \mathrm{c}+\mathrm{z} \log \mathrm{A})$ are indicated.

En general, el modelo power law presentó los mejores ajustes especie-área, que resultaron significativos $(\mathrm{P}<0.05)$, con altos valores de $\mathrm{r}^{2}$ y menores valores de AIC en las dos categorías de parches (Fig. 4). El aumento del área de los parches explicó un $82 \%$ de la variación en el aumento del número de especies. Así la riqueza también fue positivamente correlacionada con el área, explicando el $91 \%$ de la varianza. Las comparaciones post-hoc de valores-z revelaron diferencias significativas entre los dos grupos de tamaños de parches $(\mathrm{P}<0.05)$. Por otro lado, con el modelo ANCOVA se observó que la riqueza de especies varía significativamente en función del área $\left(\mathrm{F}_{1,36}=164.50, \mathrm{P}<0.0001\right)$; así como las diferencias significativas por el efecto de los tamaños de parches $\left(\mathrm{F}_{1,36}=6.48\right.$, $\mathrm{P}<0.01$ ), además de la iteración entre el área y los tamaños de parches (área: parches, $\mathrm{F}_{1,36}=$ $4.71 ; \mathrm{P}<0.01)$.

\section{DISCUSIÓN}

Los resultados de este estudio demuestran por primera vez como el tamaño de los parches de vegetación de un inselberg tropical tiene una fuerte influencia sobre la riqueza, distribución de la abundancia y composición de especies. Además, el tamaño de los parches influye fuertemente no sólo en la composición florística, sino también en la estructura de la comunidad (principales formas de vida por tamaño de parches). Así mismo, las especies fanerófitas y terófitas relacionadas con los mayores parches tal vez reflejan una mayor disponibilidad de recurso, como fue observado por Neri et al. (2016) en campos de altitudes en el sudeste de Brasil. Sin embargo, este patrón todavía no ha sido completamente demostrado para inselbergs.

A pesar del importante valor biogeográfico de los inselbergs, por tener muchas especies endémicas en pequeña escala espacial (Porembski, 2000; Porembski, 2007), además de las evidentes amenazas sobre su biodiversidad (Porembski et al., 2016), todavía son limitados los estudios que demuestren la relación de procesos ecológicos con la estructuración de las comunidades vegetales. La mayoría de estudios sobre inselbergs se han enfocado en 
la descripción general de los tipos de hábitats, comunidades vegetales y estrategias adaptativas de las especies (Barthlott et al., 1993; Lüttge, 1997; Porembski, 2007). Para la región Guayana sólo existen tres trabajos de referencia sobre caracterización general de la flora de este tipo de afloramientos rocosos (Groger, 1994; Groger, 2000; Groger \& Huber, 2007). Otros estudios han abordado análisis descriptivos sobre la relación de los tipos de comunidades vegetales sobre un complejo sistema de gradientes ambientales, principalmente por la escasez de agua, poca profundidad de los suelos y variaciones topográficas (Bremer \& Sander, 2000; Biedinger, Porembski, \& Barthlott, 2000; Lüttge, 1997; Sarthou, Kounda-Kiki, Vaçulik, Mora, \& Ponge, 2009). Sin embargo, hasta la actualidad no existe alguna investigación que implemente métodos clásicos en ecología, como los modelos SAR y SAD en diferentes comunidades vegetales de los inselbergs para comprender patrones de diversidad y establecer criterios de conservación.

En este estudio, el espacio representado por el tamaño de los parches fue considerado como un recurso dividido en puntos discretos ocupados por un individuo dentro de la comunidad (Tilman, 1994; Tilman, 1999). Por este motivo, se presume que los cambios de tamaño de los parches podría estar asociado con la disponibilidad de nutrientes y agua, como ha sido demostrado en otros ambientes de tierras secas (Rietkerk \& van de Koppel, 2008; Götzenberger et al., 2012). Este patrón ha sido observado en otros estudios, donde la cobertura de la vegetación de tierras secas es discontinua formando un mosaico de parches vegetales de gramíneas y arbustos en una matriz de suelo más o menos desnuda (Barbier, Couteron, Lejoly, Deblauwe, \& Lejeune, 2006; Rietkerk \& van de Koppel, 2008; Barbier et al., 2014). Este mosaico de parches es asumido como el resultado de un estrecha retroalimentación de la disponibilidad de recursos (suelo-agua) y de la dinámica de la vegetación (Amarasekare, 2003; Rietkerk \& van de Koppel, 2008; Meloni et al., 2017). Por otro lado, es posible que la coexistencia de las especies en estos parches sea crucial para el aumento de la riqueza de especies (Gröger \& Huber, 2007). Sin embargo, todavía sería necesario demostrar la importancia relativa de procesos dependientes de la densidad, como el balance entre facilitación y competición que puede variar considerablemente según el tamaño del parche (Matthews \& Maestre, 2012; Svejcar et al., 2015). La facilitación ha sido identificada como principal proceso responsable de la estructuración de comunidades vegetales en ambientes con fuertes filtros ambientales, como la limitación de agua, por lo tanto, cuanto mayor es el tamaño del parche más importante ha sido el proceso de facilitación (Svejcar et al., 2015; Carrión, Gastauer, Mesquita, \& Meira-Neto, 2017). Estas condiciones extremas inducen a una mayor especialización de las especies para la conservación de agua y evitación a la sequía, en comparación con otros ecosistemas alrededor, como sabanas y bosques (Barthlott et al., 1993; Porembski, 2000, Groger, 2000; Groger $\&$ Huber, 2007).

Los resultados de este estudio revelan que el patrón observado en la curva de acumulación de especies en función del área es muy similar a los patrones encontrados en otras comunidades vegetales en ambientes restringidos a pequeñas áreas, tanto en el área mínima para alcanzar más del $50 \%$ de las especies, como en la acumulación total de especies con la estabilización de la curva (Chiarucci, Viciani, Winter, \& Diekmann, 2006; Cáceres, Llambí, \& Rada, 2014; Carrión et al., 2017). El uso de curvas de rarefacción basada en individuos, en lugar de curvas basadas en área de muestreo, podría conducir a un efecto del hábitat más pronunciado, pues las curvas de rarefacción basadas en individuos tienen la ventaja de excluir los efectos de diferentes densidades (Colwell, Mao, \& Chang, 2004; Colwell et al., 2012). En este sentido, se ha discutido que la pendiente de las curvas especie-área puede ser diferente entre tipos de hábitats, como también puede ser considerablemente diferente entre áreas con características similares (Crawley \& Harral, 2001; Ricklefs \& Lovette, 1999). En este contexto, se puede suponer que la riqueza 
de especies no es sólo el resultado de la diferenciación de nichos y la diversidad de hábitats, sino también dependiente de la escala espacial (Qian, White, \& Song, 2007). En este estudio a pequeña escala, la riqueza de especies aumenta rápidamente en función del área, ya que es probable que se encuentren individuos de nuevas especies dentro de un tipo de hábitat específico. Una vez que el área es lo suficientemente grande como para contener todas las especies potencialmente detectables, la riqueza de especies aumenta con el área, ya que aumenta la probabilidad de inclusión de diferentes tipos de hábitats con diferente composición de especies (Rosenweig, 1995; Ricklefs, Qian, \& White, 2004; Matthews \& Whittaker, 2015). De allí la importancia de analizar el patrón de distribución de parches en los inselbergs.

La distribución de abundancia de especies representa un método relevante que puede contribuir en la comprobación de hipótesis sobre estructuración de las comunidades vegetales (McGill et al., 2007; Ulrich, Ollik, \& Ugland, 2010; Borda de Água et al., 2012), incluyendo comunidades vegetales en tierras secas (Ulrich et al., 2016). En este estudio, se demuestra que el modelo geométrico presentó el mejor ajuste para explicar la distribución de la abundancia de las especies en la comunidad en función del tamaño de los parches como un indicador de recursos, donde la abundancia de una especie puede ser equivalente a una proporción del espacio ocupado en relación al espacio ocupado por todas las especies de la comunidad. De esta forma, Ulrich et al. (2016) ajustando modelos log normal y serie log de comunidades vegetales de 91 sitios de tierras secas a escala global, encontraron que aproximadamente una cuarta parte de las comunidades evaluadas fueron iguales para ambos modelos. Este patrón coincide con investigaciones previas, donde los SADs pueden convertirse en funciones que explican partes contrastantes de comunidades locales (por ejemplo, Magurran \& Henderson, 2003; Vergnon, van Nees, \& Scheffer, 2012; Fattorini et al., 2016). Por un lado, las especies residentes generalmente mantienen una distribución lognormal y, por otro, las denominadas especies temporales, se describen mejor con el modelo de la serie geométrica (Magurran \& Henderson, 2003). Por esta razón, la clasificación de especies en grupos funcionales podría permitir una mejor diferenciación de patrones SADs en comunidades vegetales, incluyendo los inselbergs tropicales.

En algunos estudios se ha argumentado que la variación en la composición de especies entre los perfiles de vegetación de inselbergs tropicales está condicionada principalmente por la estructura del hábitat y el déficit hídrico (Lüttge, 1997; Porembski, 2007; Gröger \& Huber, 2007); sin embargo, no se había discutido que el tamaño de los parches de vegetación tiene un efecto significativo en la riqueza de especies, como se ha observado en este estudio. La distribuciones de tamaños de parches de vegetación han sido objeto de intenso estudio en los últimos años (Kéfi, Rietkerk, Roy, Franc, Ruiter, \& Pascual, 2011; Meloni et al., 2017), y la forma más tradicional de analizar estos patrones ha sido través de modelos especieárea. En este sentido, ha sido bien demostrado que la función potencia proporciona un buen ajuste a la distribución de tamaños de parches en diferentes condiciones ambientales y comunidades vegetales (Arrhenius, 1921; Dolnik \& Breuer, 2008). Por ejemplo, Kéfi et al. (2007) lograron demostrar que en comunidades vegetales de diferentes regiones de tierras secas la distribución de los tamaños de parches puede ser ajustada por la función potencia. Sin embargo, más recientemente se han discutido casos contrastantes donde la escala espacial puede modificar la forma de la curva de esta relación (Wilber, Kitzes, \& Harte, 2015; Pan, 2016). Por este motivo, ha surgido la necesidad de explorar con más profundidad diferentes parámetros asociados con estos modelos (por ejemplo, z y c), para explicar las causas de estos cambios. En este estudio se observó que el mayor valor de la pendiente $(\mathrm{z})$ se presenta en los parches grandes $(z=0.43)$ en comparación a los pequeños $(z=0.27)$. Pero es necesario tener cautela con estos análisis, porque las dos categorías de parches todavía se encuentran en una amplitud de área pequeña, y 
probablemente otros factores dependientes de la densidad también influyen simultáneamente. Por otra parte, también se ha discutido que los parámetros c y z están influenciados por el orden de magnitud del tamaño del área, donde un amplio ámbito de escala puede estabilizar estos parámetros (Dolnik \& Breuer, 2008; Pan, 2016). No obstante, algunos estudios también han probado que los valores de $\mathrm{z}$ pueden presentar cambios significativos en una limitada amplitud de escala espacial (Preston, 1960; Dolnik \& Breuer, 2008); por ejemplo, en tamaños de parcela muy pequeños $\left(<1 \mathrm{~m}^{2}\right)$ pueden conducir a valores z más altos (Preston 1960; Chiarucci et al., 2009).

A pesar de no haber evaluado en este estudio la heterogeneidad de los parches, con la finalidad de identificar tipos de hábitats en este inselberg de la Guayana, se presume que además del área, las características de los posibles hábitats podrían influir en la riqueza, abundancia y composición florística entre categorías de tamaños de parches. Kolasa, Manne y Pandit (2012) encontraron que la heterogeneidad de hábitat genera valores $\mathrm{z}$ dependientes de escala, mientras que el agrupamiento aleatorio de los hábitats produce valores z más robustos. Por otra parte, He y Condit (2007) observaron que las pendientes de las curvas de ocupación log$\log$ son significativamente mayores para las especies raras. Del mismo modo, las especies raras están representadas por pocos individuos en pequeñas escalas espaciales, de modo que cuanto mayor es la proporción de especies raras, mayor es la pendiente SAR (Storch, 2016). Steinmann, Eggenberg, Wohlgemuth, Linder y Zimmermann (2011) analizaron la contribución de la heterogeneidad del hábitat y el efecto del área sobre la riqueza de las especies de plantas vasculares en áreas con diferentes tamaños, encontrando que más del $70 \%$ del patrón de riqueza de especies se explica por el tamaño del área, con un efecto bastante débil de la heterogeneidad de hábitat. Adicionalmente, Ricklefs y Lovette (1999) discuten que los efectos de la heterogeneidad del hábitat son probables en taxones de hábitat especializado. En este contexto, se especula que un análisis más detallado de la distribución de abundancia de especies en función de la distribución de los tamaños de los parches y heterogeneidad de los hábitats en los inselbergs, podría proporcionar mejores explicaciones sobre los tipos de interacciones entre las especies y sobre la dinámica de parches por la cual se configuran estas comunidades vegetales.

\section{Pautas para la conservación de inse-} lbergs: Partiendo de la premisa de que las especies vegetales asociadas a los inselbergs generalmente presentan una distribución geográfica restringida con un considerable número de especies endémicas en relación al área (Barthlott et al., 1993; Porembski, 2000; Porembski, 2007), se propone el análisis simultáneo SAR y SAD como herramienta prometedora para la evaluación de patrones de diversidad en comunidades vegetales (Tjørve, 2012; Matthews \& Whittaker, 2015). En este estudio, se identificaron 12 especies endémicas de inselbergs, de las cuales Tabebuia orinocensis y Pitcairnia pruinosa se encuentran en peligro de extinción de acuerdo con los criterios de la Unión Internacional para la Conservación de la Naturaleza (IUCN) (Llamosas et al., 2003), lo que representa un importante motivo para comenzar a establecer puntos críticos de conservación. No obstante, el cambio climático e impactos antropogénicos continúan siendo una seria amenaza para la biodiversidad de los inselbergs (Porembski et al., 2016). Por esta razón, para evitar transiciones críticas, es crucial detectar señales de alerta temprana a través de estudios ecológicos. Por ejemplo, en este estudio se presume que un buen comienzo para establecer medidas de conservación, es conociendo patrones clásicos de la ecología de comunidades vegetales, como los modelos SADs y SAR en diferentes tamaños de parches y tipos de hábitats de los inselbergs.

Los resultados de esta investigación apenas representan una primera luz para entender como la distribución de los tamaños de parches pueden ser un importante predictor de riqueza, composición de especies y distribución de las abundancias con potencial aplicación en 
la biología de la conservación. Así mismo, es necesario entender a profundidad procesos subyacentes que también son determinantes en la estructura de estas comunidades vegetales, como las relaciones bióticas de las especies (facilitación y competición). Se sabe que la facilitación entre las plantas es un mecanismo importante para mantener la biodiversidad, pero todavía es fundamental explicar cómo se estructuran las comunidades en respuesta a los efectos combinados de los filtros ambientales con las interacciones bióticas. Finalmente, se considera que para los ámbitos de escala espacial limitada, los parámetros z y c podrían ser herramientas apropiadas para la descripción de los patrones de diversidad en las comunidades vegetales de los inselbergs, y que pueden contribuir al establecimiento de un sistema de referencia de curvas especie-área para una evaluación del estado de conservación de la biodiversidad. Finalmente, sugerimos la creación de nuevas áreas naturales protegidas con inselbergs, no sólo en la región Guayana, sino también en toda le región Amazónica.

\section{AGRADECIMIENTOS}

A la Organización de los Estados Americanos (OEA) por apoyar el desarrollo de esta investigación.

\section{RESUMEN}

Aunque los inselbergs son afloramientos rocosos icónicos con un alto valor biogeográfico, poco se conoce sobre los mecanismos responsables de la estructuración de comunidades vegetales. El objetivo de esta investigación fue evaluar cómo el tamaño de los parches de vegetación influye en la relación especie-área y distribución de la abundancia de especies de una comunidad en un inselberg del Monumento Natural "Piedra La Tortuga", región Guayana, Venezuela. Por este motivo, se establecieron tres preguntas de investigación: ¿Cuál es el efecto del tamaño de los parches sobre la riqueza de especies? ¿Qué tipo de modelo especie-área (SAR) presenta mejor ajuste en esos parches de vegetación? ¿Cómo es la distribución de las abundancias de las especies (SADs) es inducida por el tamaño de los parches? Se realizó un muestreo aleatorio estratificado en parches que oscilaron entre 0.34 y $14.8 \mathrm{~m}^{2}$, totalizando 40 unidades muestrales $\left(226 \mathrm{~m}^{2}\right)$. Todos los individuos encontrados en los 40 parches fueron identificados a nivel de especie. La composición florística en las diferentes muestras estuvo representada por 19 familias, 22 géneros y 24 especies, de las cuales $50 \%$ son endémicas de inselbergs y dos están amenazadas de extinción. Se identificaron dos grupos de tamaños de parches (grandes $8-15 \mathrm{~m}^{2}$ y pequeños $\leq 7.9 \mathrm{~m}^{2}$ ) en relación a la abundancia y composición de especies, con diferencias significativas entre los grupos. Las curvas de acumulación de especies para cada grupo de tamaño de parche muestran una tendencia contrastante con marcadas diferencias en la riqueza observada entre los grupos de tamaños de parches. Las curvas de los modelos SADs tuvieron un ajuste significativo de la serie geométrica en las dos categorías de parches. El modelo SAR de la función potencia presentó los mejores ajustes especie-área, donde el aumento del área de los parches explicó un $82 \%$ de la variación en el aumento del número de especies. Los resultados de este estudio demuestran por primera vez como el tamaño de los parches de vegetación de un inselberg tropical tiene una fuerte influencia sobre la riqueza, distribución de la abundancia y composición de especies. Así mismo, se determinó que el modelo geométrico SAD presentó el mejor ajuste en la comunidad en función del tamaño de los parches como un indicador de recursos, donde la abundancia de una especie puede ser equivalente a una proporción del espacio ocupado. También se presume que los cambios de tamaño de los parches, podría estar asociado con la disponibilidad de nutrientes y agua, como ha sido demostrado en otros ambientes de tierras secas. En algunos estudios se ha argumentado que la variación en la composición de especies entre los perfiles de vegetación de inselbergs tropicales está condicionada principalmente por la estructura del hábitat y el déficit hídrico. Sin embargo, no se había discutido cómo el tamaño de los parches de vegetación tiene un efecto en la riqueza. Los análisis SADs y SAR pueden proporcionar explicaciones complementarias sobre la estructuración de comunidades vegetales en inselbergs.

Palabras clave: estructura de comunidad; función potencia; parches de vegetación; rarefacción; riqueza; serie geométrica.

\section{REFERENCIAS}

Alroy, J. (2015). The hypothesis that niche space is multidimensional helps to explain how numerous species can coexist despite interacting strongly. Science Advance, 1, 1-8.

Amarasekare, P. (2003). Competitive coexistence in spatially structured environments: a synthesis. Ecology Letters, 6, 1109-1122.

Arrhenius, O. (1921). Species and area. Journal of Ecology, 9, 95-99. 
Barbier, N., Couteron, P., Lejoly, J., Deblauwe, V., \& Lejeune, O. (2006). Self-organized vegetation patterning as a fingerprint of climate and human impact on semiarid ecosystems. Journal of Ecology, 94, 537-547.

Barbier, N., Bellot, J., Couteron, P., Parsons, A. J., \& Mueller, E. N. (2014). Short-range ecogeomorphic processes in drylands systems. In E. N. Mueller, J. Wainwright, A. J. Parsons, \& L. Turnbull (Eds.), Patterns of land degradation in drylands: understanding self-organised ecogeomorphic systems (pp.85-101). Dordrecht, The Netherlands: Springer-Verlag.

Barthlott, W., Porembski, S., Szarzynski, J., \& Mund, P. (1993). Phytogeography and Vegetation of Tropical Inselbergs. In J. L. Guillaumet, M. Bein, \& H. Puig (Eds), Actes du colloque international de phytogéographie tropicale (pp. 15-24), Paris: ORSTOM editions.

Biedinger, N., Porembski, S., \& Barthlott, W. (2000). Vascular Plants on Inselbergs: Vegetative and Reproductive Strategies. In S. Porembski, \& W. Barthlott (Eds.), Inselbergs - Biotic diversity of isolated rock outcrops in tropical and temperate regions (pp. 117140). Berlin, Heidelberg: Springer-Verlag.

Borda-de-Água, L., Borges, P. A. V., Hubbell, S. P., \& Pereira, H. M. (2012). Spatial scaling of species abundance distributions. Ecography, 35, 549-556.

Bremer, H., \& Sander, H. (2000). Inselbergs: Geomorphology and Geoecology. In S. Porembski, \& W. Barthlott (Eds.), Inselbergs - Biotic diversity of isolated rock outcrops in tropical and temperate regions (pp. 7-34). Berlin, Heidelberg: Springer-Verlag.

Cáceres, Y., Llambí, L. D., \& Rada, F. (2014). Shrubs as foundation species in a high tropical alpine ecosystem: a multi-scale analysis of plant spatial interactions. Plant Ecology and Diversity, 8(2), 147-161.

Carrión, J. F., Gastauer, M., Mesquita, N., \& Meira-Neto J. A. A. (2017). Facilitation as a driver of plant assemblages in Caatinga. Journal of Arid Environments, $142,50-58$

Chao, A., Gotelli, N. J., Hsieh, T. C., Sander, E. L., Ma, K. H., Colwell, R. K., \& Aaron M. E. (2014). Rarefaction and extrapolation with Hill numbers: a framework for sampling and estimation in species diversity studies. Ecological Monographs, 84, 45-67.

Chiarucci, A., Bacaro, G., Rocchini, D., Ricotta, C., Palmer, M. W., \& Scheiner, S. M. (2009). Spatially constrained rarefaction: incorporating the autocorrelated structure of biological communities into sample-based rarefaction. Community ecology, 10(2), 209-214.

Chiarucci, A., Viciani, D., Winter, C., \& Diekmann, M. (2006). Effect of productivity on species-area curves in herbaceous vegetation: evidence of experimental and observational data. Oikos, 115, 475-483.
Colwell, R. K., Chao, A., Gotelli, N. J., Lin, S., Mao, C. X., Chazdon, R. L., \& Longino, J. T. (2012). Models and estimators linking individual-based and samplebased rarefaction, extrapolation and comparison of assemblages. Journal Plant Ecology, 5, 3-21.

Colwell, R. K., Mao, C. X., \& Chang, J. (2004). Interpolating, extrapolating, and comparing incidencebased species accumulation curves. Ecology, 85, 2717-2727.

Crawley, M. J., \& Harral, J. E. (2001). Scale dependence in plant biodiversity. Science, 291, 864-868.

Dolnik, C., \& Breuer, M. (2008). Scale dependency in the species-area relationship of plant communities. Folia Geobotanica, 43, 305-318.

Esteves, J., \& Dumith, D. A. (1997). Diversidad biológica en Amazonas: bases para una estrategia de gestión. Caracas: SADA-Amazonas-PNUD y Fundación polar.

Fattorini, S., Rigal, F., Cardoso, P., \& Borges, P. A. V. (2016). Using species abundance distribution models and diversity indices for biogeographical analyses. Acta Oecologica, 70, 21-28.

Gleason, H. A. (1922). On the relation between species and area. Ecology, 3, 158-162.

Götzenberger, L., Bello, F., Brathen, K. A., Davison, J., Dubuis, A., Guisan, A., Leps, J., Lindborg, R., Moora, M., Pärtel, M., Pellissier, L., Pottier, J., Vittoz, P., Zobel, K., \& Zobel, M. (2012). Ecological assembly rules in plant communities approaches, patterns and prospects. Biological Reviews, 87, 111-127.

Gröger, A. (1994). Análisis preliminar de la flórula y vegetación del Monumento Natural "Piedra la Tortuga", estado Amazonas, Venezuela. Acta Botánica Venezuelica, 17, 128-153.

Gröger, A. (2000). Flora and vegetation of inselbergs of Venezuelan Guayana. In S. Porembski, \& W. Barthlott (Eds.), Inselbergs - Biotic diversity of isolated rock outcrops in tropical and temperate regions (pp. 291-314). Berlin, Heidelberg: Springer-Verlag.

Gröger, A., \& Huber, O. (2007). Rock outcrop habitats in the Venezuelan Guayana lowlands: their main vegetation types and floristic components. Revista Brasileira de Botânica, 30(4), 599-609.

Guilhaumon, F., Mouillot, D., \& Gimenez, O. (2010). mmSAR: an R-package for multimodel species-area relationship inference. Ecography, 33(2), 420-424.

He, F., \& Condit, R. (2007). The distribution of species: occupancy, scale, and rarity. In D. Storch, P. Marquet, \& J. H. Brown (Eds.), Scaling Biodiversity (pp. 32-50). Cambridge: Cambridge University Press. 
Huber, O. (1995). Conservation of the Venezuelan Guayana. In J. A. Steyermark, P. E. Berry, \& B. K. Holst (Eds.), Flora of the Venezuelan Guayana (pp. 20-230). Portland, Oregon: Missouri Botanical Garden Press.

Kéfi, S., Rietkerk, M., Alados, C. L., Pueyo, Y., Papanastasis, V. P., ElAich, A., \& Ruiter, P. C. (2007). Spatial vegetation patterns and imminent desertification in Mediterranean arid ecosystems. Nature, 449, 213-217.

Kéfi, S., Rietkerk, M., Roy, M., Franc, A., Ruiter, P. C., \& Pascual, M. (2011). Robust scaling in ecosystems and the meltdown of patch size distributions before extinction. Ecology Letters, 14, 29-35.

Kolasa, J., Manne, L. L., \& Pandit, S. N. (2012). Speciesarea relationships arise from interaction of habitat heterogeneity and species pool. Hydrobiologia, 685,135-144

Llamozas, S., Stefano, R. D., Meier, W., Riina, R., Stauffer, F., Aymard, G., Huber, O., \& Ortiz, R. (2003). Libro Rojo de la Flora Venezolana. Caracas, Venezuela: Provita, Fundación Polar, Fundación Instituto Botánico de Venezuela.

Lüttge, U. (1997). Physiological ecology of tropical plants. Berlín, Heidelberg: Springer-Verlag.

Magurran, A. E. (2004). Measuring biological diversity. Oxford: Blackwell Science.

Magurran, A. E., \& Henderson, P. A. (2003). Explaining the excess of rare species innatural species abundance distributions. Nature, 422, 714-716.

Matthew, A. B., \& Maestre, F. T. (2012). Inferring local competition intensity from patch size distributions: a test using biological soil crusts. Oikos, 000, 001-009.

Matthews, T. J., Triantis, K. A., Rigal, F., Borregaard, M. K., Guilhaumon, F., \& Whittaker, R. J. (2016). Island species-area relationships and species accumulation curves are not equivalent: an analysis of habitat island datasets, Global Ecology Biogeography, 25, 607-618.

Matthews, T. J., \& Whittaker, R. J. (2015). On the species abundance distribution in applied ecology and biodiversity Management. Journal of Applied Ecology, $52(2), 443-454$.

McGill, B. J., Etienne, R. S., Gray, J. S., Alonso, D., Anderson, M. J., Benecha, H. K., ... White, E.P. (2007). Species abundance distributions: moving beyond single prediction theories to integration within an ecological framework. Ecology Letters, 10, 995-1015.

Meloni, F., Granzotti, C. R. F., Bautista, S., \& Martinez, A. S. (2017). Scale dependence and patch size distribution: clarifying patch patterns in Mediterranean drylands. Ecosphere, 8(2), 1-18.
Moreno de Las Heras, M., Saco, P. M., Willgoose, G. R., \& Tongway, D. J. (2011). Assessing landscape structure and pattern fragmentation in semiarid ecosystems using patch-size distributions. Ecological Applications, 21, 2793-2805.

Neri, A. V., Borges, G. R., Meira-Neto, J. A. V., Magnago, L. F., Trotter, I. M., Schaefer, C. E. G., \& Porembski, S. (2016). Soil and altitude drive diversity and functioning of Brazilian Páramos (campo de altitude), Journal of Plant Ecology, 10, 771-779.

Oksanen, J., Blanchet, F. G., Friendly, M., Kindt, R., Legendre, P., McGlinn, D., ... Wagner H. (2017). Vegan: Community Ecology Package. $R$ package version 2.4-3. Retrieved from https://cran.r-project. org/web/packages/vegan/vegan.pdf

Pan, X. (2013). Fundamental equations for species-area theory. Scientific Reports, 3, 1-3.

Pan, X. (2016). Application of fundamental equations to species-area theory. BMC Ecology, 16, 1-8.

Porembski, S. (2000). Biodiversity of Terrestrial Habitat Islands - the Inselbergs Evidence. In S. Porembski, \& W. Barthlott (Eds.), Inselbergs - Biotic diversity of isolated rock outcrops in tropical and temperate regions (pp. 507-513). Berlin, Heidelberg: Springer-Verlag.

Porembski, S. (2007). Tropical inselbergs: habitat types, adaptive strategies and diversity patterns. Revista Brasileira de Botânica, 30(4), 579-586.

Porembski, S., \& Barthlott, W. (2000). Granitic and Gneissic Outcrops (Inselbergs) as Centers of Diversity for Desiccation-Tolerant Vascular Plants. Plant Ecology, 151, 19-28.

Porembski, S., Silveira, F. A. O, Fiedler, L. P., Watve, A., Rabarimanarivo, M., Kouame, F., \&. Hopper, S. D. (2016). Worldwide destruction of inselbergs and related rock outcrops threatens a unique ecosystem. Biodiversity and Conservation, 25(13), 2827-2830. DOI: $10.1007 / \mathrm{s} 10531-016-1171-1$

Preston, F. W. (1960). Time and space and the variation of species. Ecology, 41, 611-627.

Qian, H., White, P. S., \& Song, J. S. (2007). Effects of regional vs. ecological factors on plant species richness: an intercontinental analysis. Ecology, 88(6), $1440-1453$

R Core Team. (2016). $R$ : A language and environment for statistical computing. R Foundation for Statistical Computing. Vienna, Austria. Retrieved from https:// www.R-project.org/

Ricklefs, R. E., \& Lovette, I. J. (1999). The role of island area per se and habitat diversity in the species-area relationship of four lesser Antillean faunal groups. Journal of Animal Ecology, 68, 1142-1160. 
Ricklefs, R. E., Qian, H., \& White, P. S. (2004). The region effect on mesoscale plant species richness between eastern Asia and eastern North America. Ecography, 27, 129-136.

Rietkerk, M., \& van de Koppel, J. (2008). Regular pattern formation in real ecosystems. Trends in Ecology and Evolution, 23, 169-175.

Rosenzweig, M. (1995). Species diversity in space and time. Cambridge: Cambridge University Press.

Sarthou, C., Kounda-Kiki, C., Vaçulik, A., Mora, P., \& Ponge, J. F. (2009). Successional patterns on tropical inselbergs: a case study on the Nouragues inselberg (French Guiana). Flora, 204, 396-407.

Sizling, A. L., Storch, D., Sizlingová, E., Reif, J., \& Gaston, K.J. (2009). Species abundance distribution results from a spatial analogy of central limit theorem. Proceedings of the National Academy of Sciences of the United States of American, 106(16), 6691-6695.

Steinmann, K., Eggenberg, S., Wohlgemuth, T., Linder, H. P., \& Zimmermann, N. E. (2011). Niches and noiseDisentangling habitat diversity and area effect on species diversity. Ecological Complexity, 8, 313-319.

Storch, D. (2016). The theory of the nested species-area relationship: geometric foundations of biodiversity scaling. Journal Vegetation Science, 27(5), 880-891.

Svejcar, L. N., Bestelmeyer, B. T., Duniway, M. C., \& James, D. K. (2015). Scale-dependent feedbacks between patch size and plant reproduction in desert grassland. Ecosystems, 18, 146-153.
Tilman, D. (1994). Competition and biodiversity in spatially structured habitats. Ecology, 75(1), 2-16.

Tilman, D. (1999). The ecological consequences of changes in biodiversity: A search for general principles. Ecology, 80 (5), 1455-1474.

Tjørve, E. (2009) Shapes and functions of species-area curves (II): a review of new models and parameterizations. Journal of Biogeography, 36, 1435-1445.

Tjørve, E. (2012). Arrhenius and Gleason revisited: new hybrid models resolve an old controversy. Journal of Biogeography, 39, 629-639.

Tjørve, E., \& Tjørve, K. M. C. (2008). The species-area relationship, self-similarity, and the true meaning of the z-value. Ecology, 89, 3528-3533.

Ulrich, W., Ollik, M., \& Ugland, K. I. (2010). A metaanalysis of species-abundance distributions. Oikos, 119, 1149-1155.

Ulrich, W., Soliveresb, S., Thomas, A. D., Dougilld, A. J., \& Maestre, F. T. (2016). Environmental correlates of species rank-abundance distributions in global drylands. Perspectives in Plant Ecology, Evolution and Systematics, 20, 56-64.

Vergnon, R., van Nees, R. H., \& Scheffer, M. (2012). Emergent neutrality leads to multimodal species abundance distributions. Nature Communication, $3,1-6$.

Wilber, M. Q., Kitzes, J., \& Harte, J. (2015). Scale collapse and the emergence of the power law species-area relationship. Global Ecology and Biogeography, 24(8), 883-895. 